\title{
DNA amplifications and aneuploidy, high proliferative activity and impaired cell cycle control characterize breast carcinomas with poor prognosis
}

\author{
Harald Blegen ${ }^{\text {a }}$, John S. Will ${ }^{\text {b }}$ B. Michael Ghadimi ${ }^{\text {b }}$, Hesed-Padilla Nash ${ }^{\text {b }}$, Anders Zetterberg ${ }^{\text {a }}$, \\ Gert Auer $^{\mathrm{a}}$ and Thomas Ried ${ }^{\mathrm{b}, *}$ \\ a Department of Oncology-Pathology, Karolinska Institute, Cancer Center Karolinska, R8:04, SE-171 76, \\ Stockholm, Sweden \\ ${ }^{\mathrm{b}}$ Genetics Department, Division of Clinical Sciences, National Cancer Institute, NIH, Building 9, Room 1N105, \\ 9 Memorial Drive, Bethesda, MD 20892, USA
}

Received 29 January 2002

Accepted 30 December 2002

\begin{abstract}
In order to explore whether specific cytogenetic abnormalities can be used to stratify tumors with a distinctly different clinical course, we performed comparative genomic hybridization $(\mathrm{CGH})$ of tumors from patients who were diagnosed with metastatic disease after an interval of less than 2 years or who remained free from distant metastases for more than 10 years. All patients presented with distant metastases after mastectomy indicating that none of the patients in this study was cured and free of remaining tumor cells. Tumors in the group of short-term survivors showed a higher average number of chromosomal copy alterations compared to the long-term survivors. Of note, the number of sub-chromosomal high-level copy number increases (amplifications) was significantly increased in the group of short-term survivors. In both short- and long-term survivors recurrent chromosomal gains were mapped to chromosomes 1q, 4q, 8q, and 5p. Copy number changes that were more frequent in the group of short-term survivors included gains of chromosome 3q, 9p, 11p and 11q and loss of 17p. Our results indicate that lowand high grade malignant breast adenocarcinomas are characterized by a specific pattern of chromosomal copy number changes. Furthermore, immunohistochemical evaluation of the expression levels of Ki-67, p2 ${ }^{\mathrm{KIP} 1}$, p21 WAF1, p53, cyclin A and cyclin E revealed a correlation between increased proliferative activity and poor outcome.
\end{abstract}

Keywords: Breast cancer, survival, $\mathrm{CGH}$, cell cycle

\section{Introduction}

Breast cancer presents itself as a heterogeneous disease with pronounced differences in the clinical course. Factors that correlate with clinical outcome include tumor stage, size and spread. However, these parameters rather describe the status quo of disease pro-

\footnotetext{
*Corresponding author: Thomas Ried, M.D., Genetics Branch, Center for Cancer Research, National Cancer Institute, NIH, Bldg. 50, Rm. 1306, 50 South Drive, Bethesda, MD 20892, USA. Tel.: +1 301594 3118; Fax: +1 301435 4428; E-mail: riedt@mail. nih.gov.
}

gression, and are less suited to predict malignancy potential in individual cases. For instance, large breast adenocarcinomas with local lymph node metastases can be observed in patients with long-term survival, whereas small tumors without detectable metastases may kill the patient shortly after diagnosis.

Previous cytogenetic and molecular analyses could correlate specific chromosomal gains or losses with tumor initiation, progression and metastatic behavior. Deletion of 1p, 7q31, 8p21-22, 16q and 17p are associated with an aggressive clinical course and a higher risk for metastatic disease [2,16,20,40,41]. Gains and amplifications of chromosome arms and bands 8q, 
17q12, 17q21-24 and 20q13 occur at higher frequency in aggressive tumors as well $[1,2,34,39,40]$. Deletion of $3 p$ and gain of chromosome 7 have been described as early aberrations [9,29] and amplification of 11q13 was found to be associated with local recurrence in node-negative breast cancer [5]. Similar results have been observed using comparative genomic hybridization (CGH) [22].

However, until today there are no conclusive data in support of a simple linear progression model of genetic events associated with tumor progression in breast cancer [24] when compared to, e.g., colorectal or cervical carcinogenesis [12,32].

Genomic instability has been suggested to play a central role for the potential of cancer cells to acquire cytogenetic aberrations and genetic mutations that are required for the multistep process of carcinogenesis. The proper orchestration of the cell cycle has emerged as a critical step for the maintenance of a diploid genome [17].

Defective cell cycle checkpoints can result in premature initiation of DNA-replication, impaired DNA repair, re-replication of DNA without cell division (polyploidisation) or a premature initiation of chromatin condensation and mitosis of incompletely replicated DNA [17]. The tumor suppressor gene p53 affects cell cycle coordination at several levels. For instance, p53 induces the expression of p21 WAF-1 [10]. This cyclin-dependent kinase inhibitor interacts with the cdk-cyclin-system in the regulation of the cell cycle. p53 plays a role for proper mitotic chromosome segregation as it is involved in the control of centrosome duplication [14]; this could explain that tumors with mutated p53 tend to have a higher degree of aneuploidy and gene amplifications $[13,35]$. Other cell cycle regulators, such as cyclin $\mathrm{E}$ and the cyclin dependent kinase inhibitor $\mathrm{p} 27^{\mathrm{KIP} 1}$ have been shown to have prognostic impact in several human malignancies, including breast carcinomas [26,27,31].

Here we explored whether genomic instability as reflected by the pattern of chromosomal aberrations, crude aneuploidy, and compromised cell cycle control can explain the striking differences in tumor progression and clinical behavior of primary breast adenocarcinomas.

\section{Materials and methods}

\subsection{Tumors}

The material comprises samples from 66 selected female patients with primary adenocarcinoma of the breast. All patients underwent radical mastectomy and none of the patients received preoperative chemo- or radiotherapy. All tumors were examined at the Department of Pathology at the Karolinska Hospital and were classified according to the World Health Organization (WHO, 1981) and graded according to Elston and Ellis [11]. All patients were selected according to the time from primary diagnosis to the time when distant metastases were detected indicating that all patients had metastatic disease at the time of operation and were not cured by the treatment. In the first group all patients presented with distant metastases more than 10 years after the primary diagnosis $(n=43)$. In the second group all patients presented with distant metastases less than 2 years from the time of diagnosis $(n=23)$. For both groups the follow-up time was sufficient to verify the final outcome. Patients diagnosed with primary adenocarcinomas in other organs (prior to or after detection of the breast carcinoma), and patients with secondary or bilateral breast tumors were excluded. None of the short-term survivors were operated on because of local recurrence whereas $47 \%$ of the long-term-survivors underwent surgery for that reason.

Of the short-term survivors 2 patients did not receive any postoperative treatment, 8 were irradiated, 4 received chemotherapy and 6 received endocrine therapy. Three patients received a combination of both irradiation and chemo- or endocrine therapy. Among the long-term survivors 4 patients received radiation therapy and one received chemotherapy. Information on treatment of loco-regional recurrence or metastatic disease was not available for any group. For a summary of the clinicopathological parameters see Table 1 and Fig. 1.

\subsection{Sample preparation}

All tumor samples were fixed in $4 \%$ phosphate buffered formaldehyde directly after the operation and paraffin embedded. From each specimen ten contiguous sections were prepared and used for immunohistochemistry (thickness $4 \mu \mathrm{m}$ ), DNA ploidy measurements $(8 \mu \mathrm{m})$ and microdissection $(50 \mu \mathrm{m})$. Tumor cell areas were microdissected or trimmed from two $50 \mu \mathrm{m}$ sections to obtain samples containing more than $80 \%$ tumor cells. Microdissected tissue was incubated overnight in $1 \mathrm{M} \mathrm{NaSCN}$ at $37^{\circ} \mathrm{C}$ and washed twice in PBS. DNA was isolated by standard proteinase $\mathrm{K}$ digestion, phenol-chloroform extraction and ethanol precipitation. 

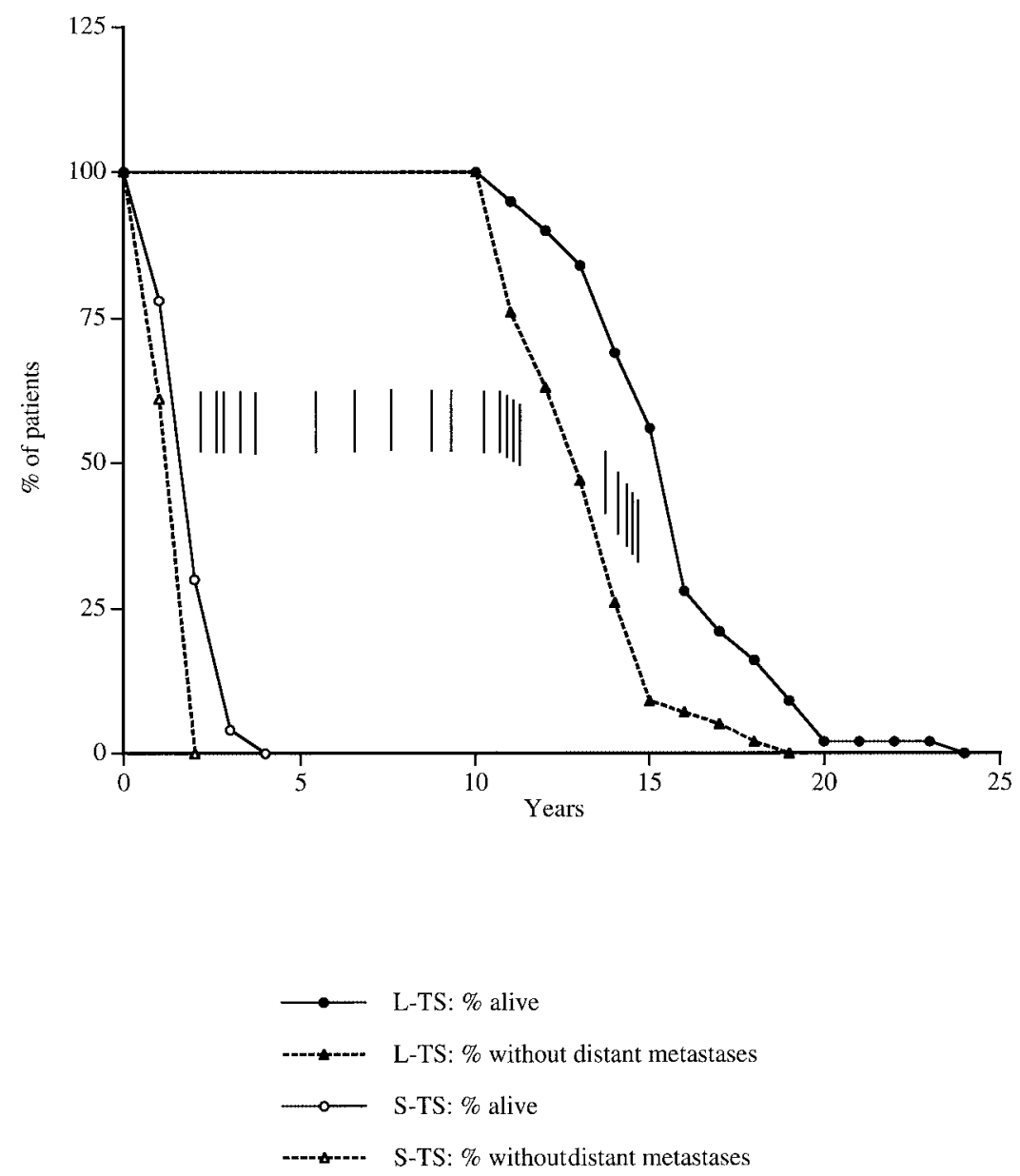

Fig. 1. Percent of patients without clinically detected distant metastases and patient survival time in years after primary diagnosis $(\mathrm{L}-\mathrm{TS}=$ long-term survivors, S-TS = short-term survivors). The vertical bars indicate the time of detection of the first loco-regional recurrence or local lymph node metastasis after the primary diagnosis in the group of L-TS.

\subsection{Immunohistochemistry}

All slides were deparaffinized with xylene, rehydrated and microwaved at $500 \mathrm{~W}$ for $2 \times 5 \mathrm{~min}$ in $10 \mathrm{mM}$ citrate buffer ( $\mathrm{pH}$ 6.0). Sections were treated with $3 \%$ hydrogen peroxide in methanol, followed by normal horse serum $(1: 20$ dilution) in $0.1 \mathrm{M}$ PBS ( $\mathrm{pH}$ 6.0) and then incubated overnight with the different antibodies diluted in $1 \%$ (wt/vol) bovine serum albumin and visualized by standard avidin biotinperoxidase complex technique (Vector Laboratories, Burlingame, CA). The antibodies used were: NCL-p27 (p27 monoclonal antibody), dilution 1:100 (Novocastra Laboratories Ltd, Newcastle upon Tyne, UK); MIB-1 (antibody against the nuclear proliferation associated antigen Ki-67, Immunotech S.A., Marseille, France), dilution 1:150; DO1 (p53 mouse mono- clonal antibody, Santa Cruz Biotechnology), dilution $1: 100$; WAF1 (Ab-1) (p21 mouse monoclonal antibody, Calbiochem), dilution 1 : 15; NCL-cyclinA (CyclinA monoclonal antibody, Novocastra Laboratories Ltd, Claremont Place, Newcastle upon Tyne, UK), dilution 1 : 100; NCL-cyclin E (Cyclin E monoclonal antibody, Novocastra Laboratories Ltd, Claremont Place, Newcastle upon Tyne, UK), dilution $1: 40$.

All slides were coded and scored independently by two pathologists. Only distinct brown nuclear staining was considered positive. The scoring system has been described previously [31].

\subsection{DNA ploidy}

Total nuclear DNA content was measured by image cytometry on Feulgen stained imprints or histo- 
Table 1

Clinicopathological characteristics of primary breast carcinomas from the selected patient groups of long-term survivors $(n=43)$ and short-term survivors $(n=23)$

\begin{tabular}{|c|c|c|}
\hline & $\begin{array}{c}\text { Long-term } \\
\text { survivors } \\
(n=43)\end{array}$ & $\begin{array}{c}\text { Short-term } \\
\text { survivors } \\
(n=23)\end{array}$ \\
\hline \multicolumn{3}{|l|}{ Type } \\
\hline Ductal & $35(82 \%)$ & $22(96 \%)$ \\
\hline Lobular & $3(7 \%)$ & 0 \\
\hline Colloid & $3(7 \%)$ & $1(4 \%)$ \\
\hline Other & $2(4 \%)$ & 0 \\
\hline \multicolumn{3}{|l|}{ Tumor size (mm) } \\
\hline Average & 20 & 31 \\
\hline Range & $7-40$ & $12-55$ \\
\hline \multicolumn{3}{|l|}{ Nodal status } \\
\hline No & $33(77 \%)$ & $11(48 \%)$ \\
\hline N1 & $10(23 \%)$ & $12(52 \%)$ \\
\hline \multicolumn{3}{|l|}{ Grade } \\
\hline I & $2(4 \%)$ & $1(4 \%)$ \\
\hline II & $24(56 \%)$ & $3(13 \%)$ \\
\hline III & $17(40 \%)$ & $19(83 \%)$ \\
\hline \multicolumn{3}{|l|}{ DNA ploidy } \\
\hline Diploid & $16(37 \%)$ & $0(0 \%)$ \\
\hline Aneuploid & $27(63 \%)$ & $23(100 \%)$ \\
\hline \multicolumn{3}{|l|}{ Average survival } \\
\hline Time (months) & 184 & 21 \\
\hline Range & $128-277$ & $2-48$ \\
\hline \multicolumn{3}{|l|}{ Primary to met. } \\
\hline Time (months) & 154 & 14 \\
\hline Range & $124-217$ & $1-24$ \\
\hline
\end{tabular}

Primary to met indicates the time from primary operation to detection of distant metastases (in months).

logical slides as described previously [37]. DNA histograms were interpreted according to a modified subjective method. The normal control cells were given the value $2 c$, denoting the normal diploid DNA content and all tumor-cell DNA values were expressed in relation to that. The histograms were divided into two groups. Cases with a major peak near the $2 \mathrm{c}$ region $(1.5 c-2.5 c)$ and $<10 \%$ cells exceeding $2.5 c$ were denoted diploid. DNA profiles with a stemline outside the diploid region and distinctly scattered DNA values exceeding the tetraploid region $(3.5 \mathrm{c}-4.5 \mathrm{c})$ were classified as aneuploid.

\subsection{Comparative Genomic Hybridization (CGH)}

CGH was performed on normal, sex-matched metaphase chromosomes prepared according to standard procedures. Control DNA was labeled with digoxigenin dUTP (Boehringer Mannheim) by nick-translation. Tumor DNA was extracted using proteinase $\mathrm{K}$ digestion and phenol extraction. Labeling of genomic tumor DNA was performed by nick translation substituting dTTP by biotin-16-dUTP (Boehringer Mannheim). $500 \mathrm{ng}$ of each, differentially labeled genomic DNA was precipitated together with an excess $(30 \mu \mathrm{g})$ of the Cot-1 fraction of human DNA (Gibco BRL, Gaithersburg, MD). The probe DNA was resuspended in $10 \mu \mathrm{l}$ of hybridization solution (50\% formamide, $2 \times$ SSC, $10 \%$ dextran sulfate), denatured (5 minutes, $75^{\circ} \mathrm{C}$ ), and preannealed for 1 hour at $37^{\circ} \mathrm{C}$. The normal metaphase chromosomes were denatured separately $(70 \%$ formamide, $2 \times \mathrm{SSC})$ for 2 minutes at $75^{\circ} \mathrm{C}$, and dehydrated through an ethanol series. Hybridization took place under a coverslip for 2-4 days at $37^{\circ} \mathrm{C}$. Posthybridization washes and immunocytochemical detection was performed as described [21]. Biotin labeled tumor sequences were detected with avidin conjugated to FITC (Vector laboratories), and the digoxigenin labeled reference DNA was developed using a mouse anti-digoxin antibody, followed by a TRITC conjugated anti-mouse antibody (Sigma). The slides were counterstained with DAPI and embedded in an antifade solution containing para-phenylenediamine (Sigma).

Gray level images were acquired for each fluorochrome with a cooled CCD camera (Sensys, Photometrics, Tucson, AZ) coupled to a Leica DMRXA epifluorescence microscope using sequential exposure through fluorochrome specific filters (TR1, TR2, TR3, Cy5; Chroma Technology, Brattleboro, VT) using the Leica Q-FISH ACAPS imaging system. Chromosomes were identified using DAPI-banding and cohybridization with centromere specific DNA-probes. Fluorescence ratio images were calculated with the Cytovision CGH software (Applied Imaging, Ltd., Tyne \& Wear, UK). Average ratio profiles were calculated from at least 8 cells. Average ratio profiles were the basis for the evaluation of copy number changes in all cases.

The average number of copy alterations (ANCA) was calculated by adding all chromosomal events for different groups divided by the number of cases in each group. The average number regional amplifications (ANRA) was calculated by adding all regional amplifications for different groups divided by the number of cases in each group. 


\section{Results}

\subsection{DNA ploidy}

Among the long-term survivors $(n=43) 16$ tumors were near-diploid (37\%) (below referred to as diploid) and 23 tumors were highly aneuploid (53\%). In 5 cases ploidy levels could not be reliably measured. $35 \%$ of the tumors from patients in the group that was diagnosed with local lymph node metastases or local recurrences after the primary diagnosis were diploid and $55 \%$ were aneuploid. In only one case did the ploidy value differ between the primary tumor and the recurrence, with the primary tumor being diploid and the loco-regional recurrence 14 years later aneuploid. All tumors in the group of short-term survivors $(n=23)$ were aneuploid.

\subsection{Immunohistochemistry}

Long-term survivors: The primary tumors in the group of long-term survivors were characterized by a low proliferative activity as reflected by low numbers of tumor cells expressing Ki-67 $(<20 \%$ positive cells in $68 \%$ of the cases) and cyclin A ( $<5 \%$ positive cells in $53 \%$ of the cases) and a high number of cells expressing p27 ( $>50 \%$ positive cells detected in $79 \%$ of the cases). Positive p53 staining was infrequent and p53 positivity together with undetectable p21 in the same tumors was found in only $12 \%$ of the cases (Table 2). All tumors revealed a low cyclin E staining pattern $(<1 \%$ positive cells/case). Among the patients who were diagnosed with loco-regional recurrence a moderate increase in MIB-1 was found in only $15 \%$ of the primary tumors. Increased levels of cyclin A $(>5 \%$ positive tumor cells) were revealed in $40 \%$ (with recurrence) and 52\% (without recurrence). Regarding p27 there were no differences in staining patterns between primary tumors from patients with or without local recurrence.

Short-term survivors: The tumors from the group of short-term survivors were characterized by moderately (20-50\% positive cells) or highly ( $>50 \%$ positive cells) increased MIB-1 staining in $74 \%$ of the cases, overexpression ( $>5 \%$ positive cells) of cyclin A in $87 \%$ of the cases and low expression $(<50 \%$ positive cells) of $\mathrm{p} 27^{\mathrm{KIP} 1}$ in $74 \%$ of the cases. Positive p53 staining together with undetectable p21 in the same tumors were found in $30 \%$ of the cases (see Table 2). Furthermore, $26 \%$ of these tumors revealed overexpression ( $>10 \%$ positive cells/case) of cyclin E. None of these patients were diagnosed with loco-regional recurrence.
Table 2

Immunohistochemical characteristics of primary breast carcinomas from the selected patient groups of long-term survivors $(n=43)$ and short-term survivors $(n=23)$

\begin{tabular}{ccc}
\hline & $\begin{array}{c}\text { Long-term } \\
\text { survivors }(n=43)\end{array}$ & $\begin{array}{c}\text { Short-term } \\
\text { survivors }(n=23)\end{array}$ \\
\hline $\begin{array}{c}\text { MIB-1 } \\
\leqslant 20 \% \\
>20 \%\end{array}$ & $29(67 \%)$ & $6(26 \%)$ \\
cyclin A & $14(33 \%)$ & $17(74 \%)$ \\
$\leqslant 5 \%$ & & \\
$>5 \%$ & $23(53 \%)$ & $3(13 \%)$ \\
p27KIP1 & $20(47 \%)$ & $20(87 \%)$ \\
$\leqslant 50 \%$ & & $17(74 \%)$ \\
$>50 \%$ & $9(21 \%)$ & $6(26 \%)$ \\
p53/p21 & $34(79 \%)$ & $7(30 \%)$ \\
pattern $+/-$ & $5(12 \%)$ & $16(70 \%)$ \\
others & $38(88 \%)$ & \\
\hline
\end{tabular}

Pattern $+/-$ refers to positive staining of p53 and undetectable p21 staining in the same tumor.

\subsection{Comparative genomic hybridization}

Thirty-five tumors could be analyzed by CGH. The remaining cases revealed only insufficient DNA or DNA of poor quality. CGH revealed a different pattern of chromosomal copy number changes in primary breast carcinomas when long-term survivors $(n=17)$ and short-term survivors ( $n=18$ ) were compared. The most significant differences are shown in Fig. 2. For both groups a relatively high number of chromosomal copy number changes was observed (Fig. 3).

Among the long-term survivors the average number of copy alterations (ANCA) was 8.7 and the average number of regional amplifications (ANRA) was 0.4. Diploid tumors from this group of patients revealed an ANCA of 5.7 and an ANRA value of zero (i.e., no amplified regions). The ANCA value of the aneuploid tumors from the same group was 10.1 and the ANRA value 0.6. The ANRA value revealed the most pronounced difference when the long-term survivors were divided into diploid and aneuploid tumors. Differences in tumor grade, expression levels of MIB-1, cyclin A, p53, p21 and p27 were not significant when compared with the ANCA and ANRA values.

The most frequent chromosomal aberrations in primary tumors from long-term survivors were gains of chromosome 5p (71\%), 4q (59\%), 6q (53\%), Xq (47\%), $1 \mathrm{q}(28 \%), 8 \mathrm{q}(41 \%)$ and $13 \mathrm{q}(41 \%)$. Gain of $20 \mathrm{q}$ was found in only one case. The most frequent losses were mapped to chromosome arms 16q (29\%), 17p (23\%) and $22 \mathrm{q}(18 \%)$. 


\begin{abstract}
A) $n=17$
\end{abstract}

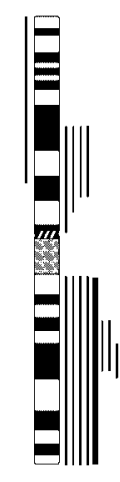

1

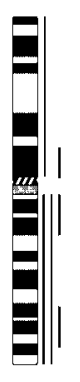

3

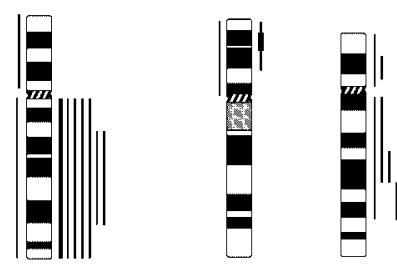

12
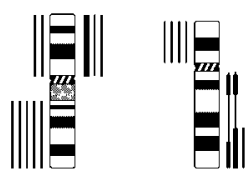

16

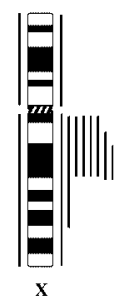

B) $\mathrm{n}=18$

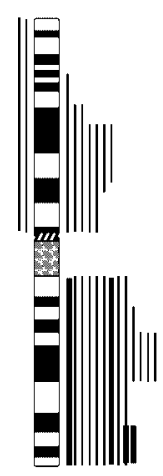

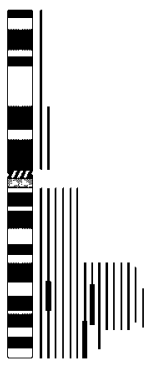

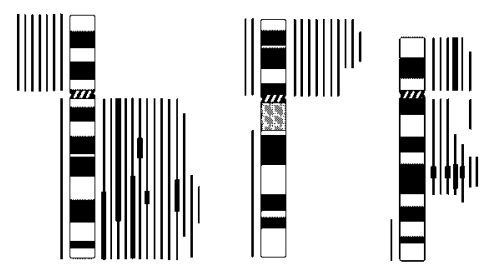

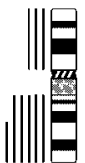

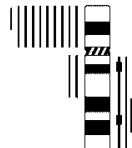

17

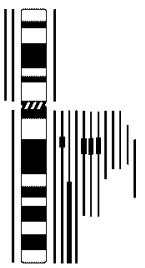

$\mathrm{x}$

Fig. 2. The most pronounced differences in chromosomal rearrangements in primary breast carcinomas from long-term survivors (A) and short-term survivors (B). Bars on the right side of the chromosome ideogram indicate gains and bars on the left side indicate loss of chromosomal material. High copy number alterations are marked by bold bars.

In the group of patients that were diagnosed with either loco-regional recurrence or local lymph node metastases between 3-17 years after the primary diagnosis the ANCA and ANRA values of the primary tumors were 9.4 and 0.6 , respectively. In those without recurrence the ANCA value was 7.0 and the ANRA value was 0.3 .

We also investigated 14 of the loco-regional recurrences by CGH. In these cases the average time from primary diagnosis to the first recurrence was 105 months (range 35-204). The ANCA value for the recurrent tumors was 8.9 and the ANRA value 0.5. Of these tumors three were found to have gains or ampli- fications that mapped to 20q. A summary of the most significant differences of chromosomal aberrations between the primary tumors and the recurrences is shown in Fig. 4.

Despite the fact that the ANCA values were similar for the two groups a pronounced difference was seen between the primary tumors and the recurrences regarding the average number of high copy number gains of whole chromosome arms. For the primary tumors the average number of high copy number gains of whole chromosome arms was 0.1 whereas for the recurrences it was 0.9 (Fig. 5). Figure 6A-D shows the CGH pattern of two representative cases with progres- 

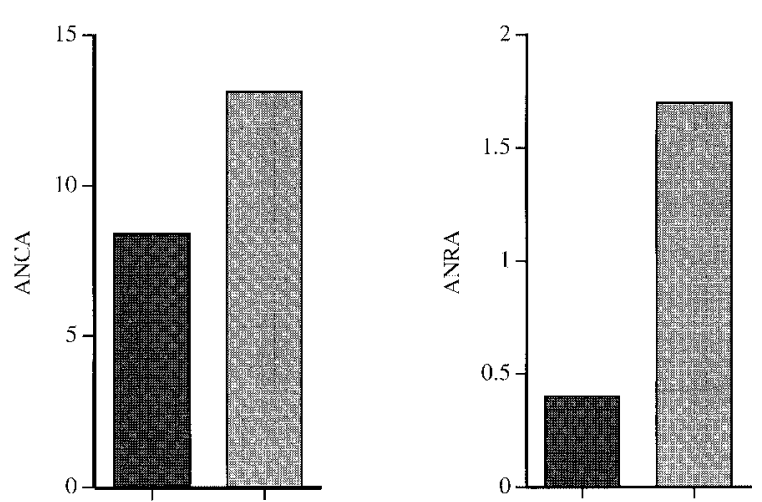

Fig. 3. (A) Average number of chromosomal aberrations (ANCA) and (B) average number of regional amplifications (ANRA) in primary breast carcinomas from patients with short- and long-term survival.

sion from primary tumor to recurrences/local lymph node metastases.

Breast carcinomas from the short-term survivors revealed a high level of genomic instability. All tumors from this group of patients were aneuploid and the ANCA value was 13.1 and the ANRA value 1.7. The most frequent chromosomal aberrations in tumors from short-term survivors were gains of $3 \mathrm{q}(83 \%)$, 1q (78\%), 8q (78\%), 4q (67\%), Xq (67\%), 5p (61\%), $13 \mathrm{q}(55 \%)$ and $6 \mathrm{q}(55 \%)$. Gain of $20 \mathrm{q}$ was found in only one case. The most frequent losses were mapped to chromosome arms 17p (55\%), 16q (29\%), $18 \mathrm{q}(22 \%)$ and $22 \mathrm{q}(22 \%)$. Similar to the primary tumors of the long-term survivors, high copy number gains of whole chromosome arms was infrequently seen. Regional copy number alterations found in more than one tumor were mapped to chromosome bands 1q41-44, 3q25-26, 8q22-23, 12q15-21, 17q23-24 and $\mathrm{Xq} 21$. No amplification was seen on chromosome arm $20 \mathrm{q}$ or $11 \mathrm{q} 13$, the mapping position of the AIBnuclear co-receptor gene and cyclin D1, respectively.

Similar to the group of long-term survivors, overexpression of cyclin A, cyclin E, MIB-1, p27 or p21 was not associated with specific alterations of the ANCA or ANRA values. However, in cases that revealed p53 positive staining pattern indicating overexpression of a mutated version of this tumor suppressor gene, both the

ANCA (14.5) and the ANRA (2.1) values were clearly increased.

\section{Discussion}

The integrity of the genome is maintained through the coordinated expression of genes that are involved in the control of cell cycle progression (reviewed in $[18,30])$. The inactivation or functional impairment of these cell cycle regulators is a key event during carcinogenesis. The emerging genetic instability may be a prerequisite for the acquisition of chromosomal aberrations and gene mutations that are specific and recurring events in cancer. We hypothesized that the strikingly different clinical course breast cancer can take may be attributed to specific alterations in cell cycle control, and, possibly, is reflected by a specific pattern of chromosomal gains and losses and aneuploidy.

We have therefore selected two clinically different groups of patients with primary breast carcinomas who succumbed to the disease either shortly after diagnosis or after a long interval. The patients in both groups, however, developed distant metastases a few months to years before death, and were therefore not cured at time of surgery.

In the group of long-term survivors traditional prognostic markers such as tumor size, grade, ploidy, Sphase and clinical stage may indicate that these are low grade malignant tumors and that surgery would be the single treatment of choice. In our selected material the primary tumors had an average size of $20 \mathrm{~mm}, 82 \%$ were ductal, $40 \%$ grade 3 , and $23 \%$ had local ipsilateral lymph node involvement at the time of primary diagnosis (of those, $30 \%$ had $>3$ nodes affected) (see Table 1). The fact that $30-50 \%$ of those patients survived between 17-27 years after surgery despite having axillary lymph node metastases may indicate that axillary lymph node status is not sensitive enough to predict prognosis in the individual case [25].

In the group of long-term survivors $35 \%(15 / 43)$ of the patients received postoperative radiotherapy and only 5\% (2/43) patients received chemotherapy. Previous studies of long-term survivors have shown that local recurrence can be prevented by postoperative radiotherapy. However, this treatment has only minor impact on disease specific or overall survival $[8,19]$. Our results support this observation. In the group of patients treated with radiotherapy we found a reduction in the numbers of loco-regional recurrences and late local lymph node metastases. We noted a slight 


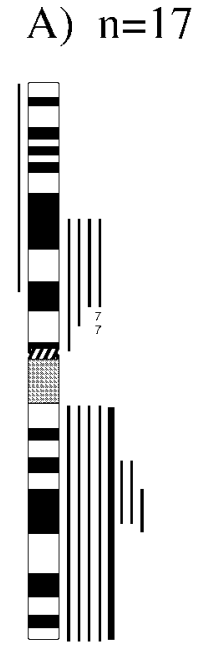

1

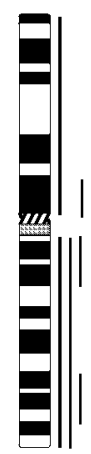

3

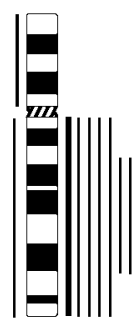

8

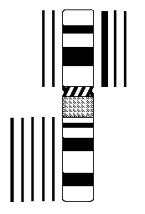

16

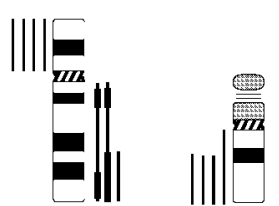

17

22

B) $n=14$

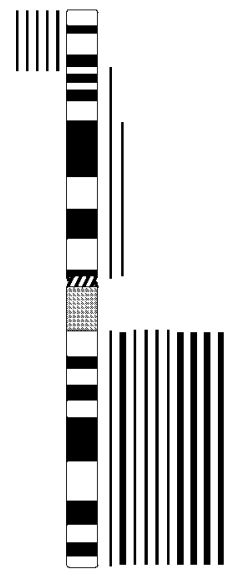

1

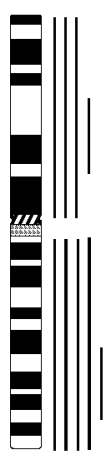

3

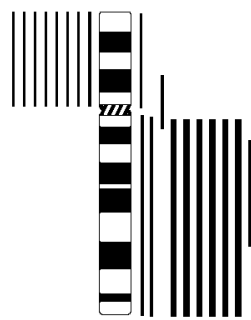

8

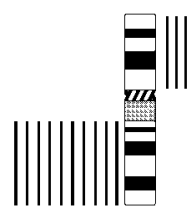

16

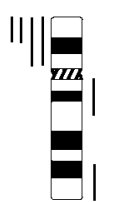

17

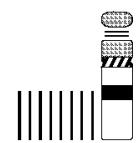

22

Fig. 4. Karyogram of chromosomal gains and losses in primary breast carcinomas (A) and in loco-regional recurrences/lymph node metastases (B) in patients with long-term survival. Bars on the right side of the chromosome ideogram indicate gains and bars on the left side indicate loss of genetic material. Solid bars denote high level copy number increases (amplifications).

delay from the time of surgery to the detection of clinically manifest metastases and survival in patients that received radiotherapy (140 vs. 161 months) or patients that were treated with surgery alone (170 vs. 189 months). This delay may reflect that radiotherapy was chosen for tumors that appeared to be clinically more aggressive, and that this assumption was correct [6].

In the group of short-term survivors the average tumor size was larger $(31 \mathrm{~mm}), 96 \%$ were ductal, $83 \%$ grade 3 and $52 \%$ had local lymph node involvement at the time of primary diagnosis (of those, $83 \%$ had $>3$ positive nodes). This group of patients was correctly assumed to have a more aggressive disease, which is also reflected by a more aggressive treatment strategy towards these patients as described in Materials and methods. However, even with intensified, albeit heterogeneous treatment schedules these patients rapidly developed clinically manifest distant metastases and died from the disease. 


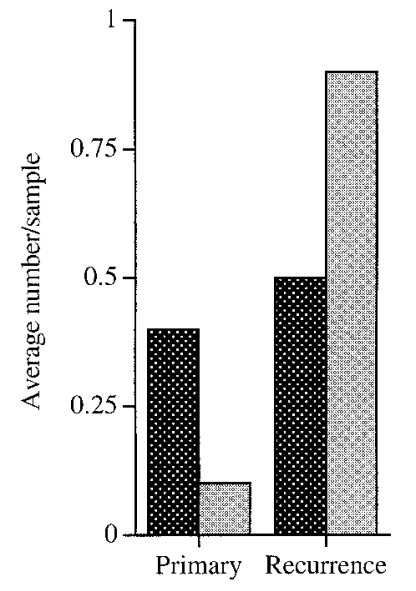

Average number of regional amplifications

Average number of high copy number alterations of whole chromosomes /chromosome arms

Fig. 5. CGH data from primary carcinomas and local recurrencies. Average number of regional amplification (ANRA) and average number of high copy number gains of whole chromosome/chromosome arms in breast carcinomas from patients with long-term survival.

In this study of breast carcinomas we found that tumors from long-term survivors in general have low proliferative activity, display normal p53 and p 21 WAF-1 levels and express high levels of the cell cycle inhibitor p2 $7^{\mathrm{KIP} 1}$ and low levels of cyclin E. Regarding the ploidy levels primary tumors from the longterm survivors seemed to be a heterogeneous group. However, the aneuploid cases were found with similar frequency in the subgroups of tumors revealing high or low cyclin A, loco-regional recurrence or not and high or low histological grading. Among tumors overexpressing p53 50\% were aneuploid whereas for p53 "negative" cases $64 \%$ were aneuploid. Among the diploid and aneuploid cases there was a homogenous distribution of cases overexpressing cyclin A, revealing elevated proliferation rates, low levels of $\mathrm{p} 27^{\mathrm{KIP} 1}$ and overexpression of p53.

Breast carcinomas from the short-term survivors were all aneuploid, had in general a high proliferation activity, overexpressed cyclin A and sometimes cyclin E or both, showed low expression levels of $\mathrm{p} 27^{\mathrm{KIP} 1}$ and signs of p53 abnormalities. Previous studies have shown that cyclin A is associated with reduced survival in breast cancer patients $[3,4]$. This immunohis- tochemical profile of the aggressive tumors supports previous studies on these cell cycle regulatory proteins in breast carcinomas with poor outcome [23,28,31,38]. The large number of tumors overexpressing cyclin $\mathrm{A}$ is in contrast to a previous study [7].

Despite infrequent and unspecific alterations in the expression pattern of cell cycle regulatory proteins, tumors from the long-term survivors generally reveal a high number of chromosomal aberrations (ANCA value of 8 and ANRA value of 0.4 ). The diploid carcinomas in this group had lower ANCA and ANRA values than the aneuploid tumors (5.7/10.1, and 0.0/0.6, respectively). These values are higher than the ones in a previous study from our laboratory [15]. In this study, however, tumor DNA was extracted from tissue blocks without enrichment by microdissection. Therefore, dilution with normal cells could possibly explain the higher number of aberrations found in the present study.

The ANCA value of the primary tumors from shortterm survivors was 13.1 and the ANRA value was 1.7. Among tumors with increased numbers of p53 positive cells the ANCA value was 14.5 and the ANRA value 2.1. In a recent study of ovarian carcinomas from our group a similar correlation was seen between high ANRA value and overexpression of p53 or cyclin A (submitted for publication). As discussed above most of these aggressive breast carcinomas revealed a high malignancy grade, high Ki-67 expression and frequently inappropriate expression patterns of cyclin A, cyclin E or $\mathrm{p} 27^{\mathrm{KIP} 1}$, suggesting a disturbed cell cycle control.

Comparing specific chromosomal gains and losses in tumors from the long- and short-term survivors notable differences became evident (Fig. 3). Gain of 13q has not been reported from primary breast carcinomas in previous studies using CGH. However, in a study of colorectal tumors we found gain of $13 q$ in $50 \%$ of invasive carcinomas, frequently with high copy number alterations [33]. Similar results have also been published from breast cancer cell lines [15] and endometrial carcinoma [36]. In two cases we found amplifications which mapped to the minimal region $13 \mathrm{q} 21$. This could indicate that yet an unidentified oncogene(s) map to this region.

The amplified regions on 12q15-21 and Xq21 have to our knowledge not previously been reported and would be highly interesting regions in the search for tumor associated genes especially since they were only found in tumors from the short-term survivors.

As a model for the development of aggressive tumors that show high levels of MIB1 and low levels of 

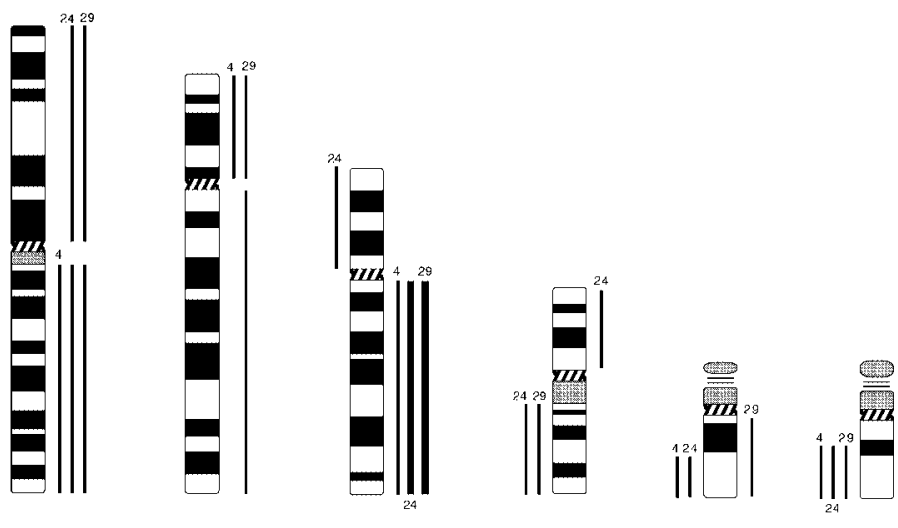

1

3

5

16

21

B)

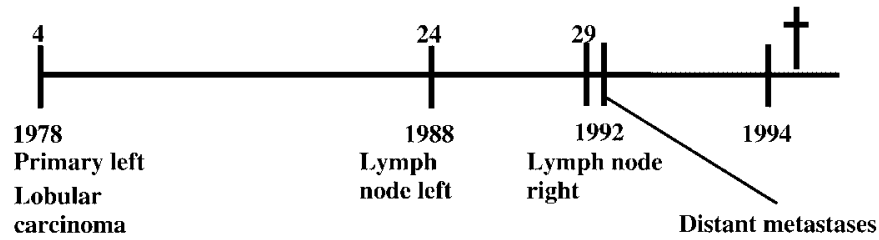

C)

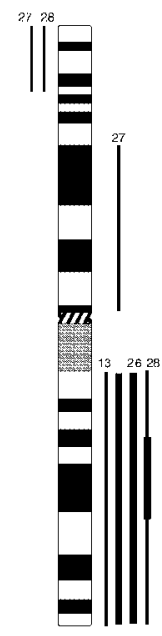

1

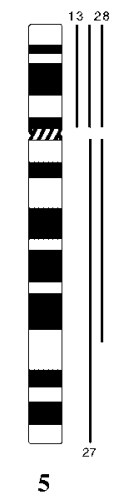

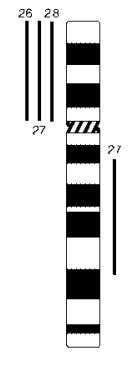

8

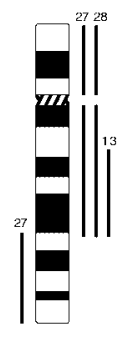

12

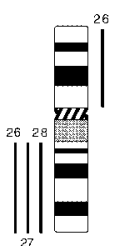

16

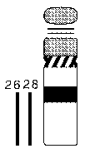

22

D)

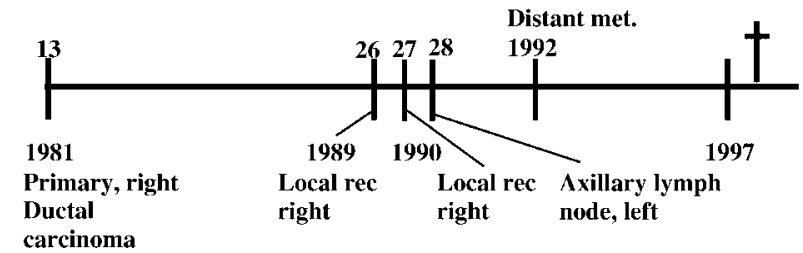

Fig. 6. Two representative cases of breast carcinoma with primary tumors 4 and 13 (indicated in (B) and (D)) and their recurrencies. In (A) and (C) specific and recurrent chromosomal gains and losses are shown for each tumor as indicated by numbers on top of the bars. 
p $27^{\mathrm{KIP} 1}$ we suggest that increased proliferative activity and impaired cell cycle control renders the tumor an increased propensity to acquire a cytogenetic aberration pattern that is required for rapid disease progression. Notably, copy number changes on, e.g., chromosome 3 and loss on, e.g., chromosome 17 seem to correlate with rapid development of metastatic disease, that, in turn is tightly correlated to patient death.

\section{Acknowledgements}

We are grateful to Ulla Aspenblad and Inga Maurin for excellent technical assistance and to Buddy Chen for manuscript editing. This work was supported by grants from the Swedish Cancer Society and the Cancer Society in Stockholm (to GA, AZ and HB), by grants from the Swedish Medical Research Foundation and Lars Hiertas Minnesstiftelse (to HB) and by the Deutsche Krebshilfe (to BMG).

\section{References}

[1] M. Barlund, M. Tirkkonen, F. Forozan, M.M. Tanner, O. Kallioniemi and A. Kallioniemi, Increased copy number at 17q22-q24 by CGH in breast cancer is due to high-level amplification of two separate regions, Genes Chromosomes Cancer 20(4) (1997), 372-376.

[2] I. Bieche, M.H. Champeme, F. Matifas, K. Hacene, R. Callahan and R. Lidereau, Loss of heterozygosity on chromosome 7q and aggressive primary breast cancer, Lancet 339(8786) (1992), 139-143.

[3] I.R. Bukholm, G. Bukholm and J.M. Nesland, Over-expression of cyclin A is highly associated with early relapse and reduced survival in patients with primary breast carcinomas, Int. J. Cancer 93(2) (2001), 283-287.

[4] I.R. Bukholm, G. Bukholm and J.M. Nesland, Coexpression of cyclin A and beta-catenin and survival in breast cancer patients, Int. J. Cancer 94(1) (2001), 148-149.

[5] M.H. Champeme, I. Bieche, S. Lizard and R. Lidereau, 11q13 amplification in local recurrence of human primary breast cancer, Genes Chromosomes Cancer 12(2) (1995), 128-133.

[6] M.A. Chaudary, D. Tong, R. Millis, P. Smith, I.S. Fentiman and R.D. Rubens, Loco-regional recurrence following mastectomy for early breast carcinoma: efficacy of radiotherapy at the time of recurrence, Eur. J. Surg. Oncol. 23(4) (1997), 348-353.

[7] F. Courjal, G. Louason, P. Speiser, D. Katsaros, R. Zeillinger and $\mathrm{C}$. Theillet, Cyclin gene amplification and overexpression in breast and ovarian cancers: evidence for the selection of cyclin D1 in breast and cyclin E in ovarian tumors, Int. J. Cancer 69(4) (1996), 247-253.

[8] J. Cuzick, H. Stewart, L. Rutqvist, J. Houghton, R. Edwards, C. Redmond, R. Peto, M. Baum, B. Fisher, H. Host et al., Cause-specific mortality in long-term survivors of breast cancer who participated in trials of radiotherapy, J. Clin. Oncol. 12(3) (1994), 447-453.
[9] G. Deng, Y. Lu, G. Zlotnikov, A.D. Thor and H.S. Smith, Loss of heterozygosity in normal tissue adjacent to breast carcinomas, Science 274(5295) (1996), 2057-2059.

[10] W.S. el-Deiry, J.W. Harper, P.M. O’Connor, V.E. Velculescu, C.E. Canman, J. Jackman, J.A. Pietenpol, M. Burrell, D.E. Hill, Y. Wang et al., WAF1/CIP1 is induced in p53-mediated G1 arrest and apoptosis, Cancer Res. 54(5) (1994), 1169-1174.

[11] C.W. Elston and I.O. Ellis, Pathological prognostic factors in breast cancer. I. The value of histological grade in breast cancer: experience from a large study with long-term follow-up, Histopathology 19 (1991), 403-410, Histopathology 41(3A) (2002), 151.

[12] E.R. Fearon and B. Vogelstein, A genetic model for colorectal tumorigenesis, Cell 61(5) (1990), 759-767.

[13] K. Fukasawa, F. Wiener, G.F. Vande Woude and S. Mai, Genomic instability and apoptosis are frequent in p53 deficient young mice, Oncogene 15(11) (1997), 1295-1302.

[14] K. Fukasawa, T. Choi, R. Kuriyama, S. Rulong and G.F. Vande Woude, Abnormal centrosome amplification in the absence of p53, Science 271(5256) (1996), 1744-1747.

[15] X.Y. Guan, P.S. Meltzer, W.S. Dalton and J.M. Trent, Identification of cryptic sites of DNA sequence amplification in human breast cancer by chromosome microdissection, Nat. Genet. 8(2) (1994), 155-161.

[16] P.J. Hainsworth, K.L. Raphael, R.G. Stillwell, R.C. Bennett and O.M. Garson, Rearrangement of chromosome 1p in breast cancer correlates with poor prognostic features, Br. J. Cancer $\mathbf{6 6}(1)$ (1992), 131-135.

[17] L. Hartwell, T. Weinert, L. Kadyk and B. Garvik, Cell cycle checkpoints, genomic integrity, and cancer, Cold Spring Harb. Symp. Quant. Biol. 59 (1994), 259-263.

[18] L. Hartwell, Defects in a cell cycle checkpoint may be responsible for the genomic instability of cancer cells, Cell 71(4) (1992), 543-546.

[19] J.L. Haybittle, D. Brinkley, J. Houghton, R.P. A'Hern and M. Baum, Postoperative radiotherapy and late mortality: evidence from the Cancer Research Campaign trial for early breast cancer, BMJ 298(6688) (1989), 1611-1614.

[20] S. Heim and F. Mitelman, Cancer Cytogenetics, Wiley-Liss, 1995.

[21] K. Heselmeyer, E. Schrock, S. du Manoir, H. Blegen, K. Shah, R. Steinbeck, G. Auer and T. Ried, Gain of chromosome 3q defines the transition from severe dysplasia to invasive carcinoma of the uterine cervix, Proc. Natl. Acad. Sci. USA 93(1) (1996), $479-484$.

[22] J.J. Isola, O.P. Kallioniemi, L.W. Chu, S.A. Fuqua, S.G. Hilsenbeck, C.K. Osborne and F.M. Waldman, Genetic aberrations detected by comparative genomic hybridization predict outcome in node-negative breast cancer, Am. J. Pathol. 147(4) (1995), 905-911.

[23] T. Jansson, M. Inganas, S. Sjogren, T. Norberg, A. Lindgren, L. Holmberg and J. Bergh, p53 Status predicts survival in breast cancer patients treated with or without postoperative radiotherapy: a novel hypothesis based on clinical findings, J. Clin. Oncol. 13(11) (1995), 2745-2751.

[24] T. Kuukasjarvi, M. Tanner, S. Pennanen, R. Karhu, O.P. Kallioniemi and J. Isola, Genetic changes in intraductal breast cancer detected by comparative genomic hybridization, Am. J. Pathol. 150(4) (1997), 1465-1471. 
[25] F. Linell and F. Rank, Breast cancer. Comments on histologic classifications with reference to histogenesis and prognosis, Universitetsförlaget Dialogos, Lund, 1989.

[26] M. Loda, B. Cukor, S.W. Tam, P. Lavin, M. Fiorentino, G.F. Draetta, J.M. Jessup and M. Pagano, Increased proteasomedependent degradation of the cyclin-dependent kinase inhibitor p27 in aggressive colorectal carcinomas, Nat. Med. 3(2) (1997), 231-234.

[27] E.W. Newcomb, M. Sosnow, R.I. Demopoulos, A. ZeleniuchJacquotte, J. Sorich and J.L. Speyer, Expression of the cell cycle inhibitor p27KIP1 is a new prognostic marker associated with survival in epithelial ovarian tumors, Am. J. Pathol. 154(1) (1999), 119-125.

[28] T. Norberg, T. Jansson, S. Sjogren, C. Martensson, I. Andreasson, M.L. Fjallskog, H. Lindman, H. Nordgren, A. Lindgren, L. Holmberg and J. Bergh, Overview on human breast cancer with focus on prognostic and predictive factors with special attention on the tumour suppressor gene p53, Acta Oncol. 35(Suppl. 5) (1996), 96-102.

[29] N. Pandis, S. Heim, G. Bardi, I. Idvall, N. Mandahl and F. Mitelman, Chromosome analysis of 20 breast carcinomas: cytogenetic multiclonality and karyotypic-pathologic correlations, Genes Chromosomes Cancer 6(1) (1993), 51-57.

[30] A.G. Paulovich, D.P. Toczyski and L.H. Hartwell, When checkpoints fail, Cell 88(3) (1997), 315-321.

[31] P.L. Porter, K.E. Malone, P.J. Heagerty, G.M. Alexander, L.A. Gatti, E.J. Firpo, J.R. Daling and J.M. Roberts, Expression of cell-cycle regulators $\mathrm{p} 27 \mathrm{Kip} 1$ and cyclin $\mathrm{E}$, alone and in combination, correlate with survival in young breast cancer patients, Nat. Med. 3(2) (1997), 222-225.

[32] T. Ried, K. Heselmeyer-Haddad, H. Blegen, E. Schrock and G. Auer, Genomic changes defining the genesis, progression, and malignancy potential in solid human tumors: a phenotype/genotype correlation, Genes Chromosomes Cancer 25(3) (1999), 195-204.

[33] T. Ried, R. Knutzen, R. Steinbeck, H. Blegen, E. Schrock, K. Heselmeyer, S. du Manoir and G. Auer, Comparative genomic hybridization reveals a specific pattern of chromosomal gains and losses during the genesis of colorectal tumors, Genes Chromosomes Cancer 15(4) (1996), 234-245.

[34] D.J. Slamon, W. Godolphin, L.A. Jones, J.A. Holt, S.G. Wong, D.E. Keith, W.J. Levin, S.G. Stuart, J. Udove, A. Ullrich et al., Studies of the HER-2/neu proto-oncogene in human breast and ovarian cancer, Science 244(4905) (1989), 707-712.

[35] M.L. Smith and A.J. Fornace, Jr., Genomic instability and the role of p53 mutations in cancer cells, Curr. Opin. Oncol. 7(1) (1995), 69-75.

[36] G. Sonoda, S. du Manoir, A.K. Godwin, D.W. Bell, Z. Liu, M. Hogan, M. Yakushiji and J.R. Testa, Detection of DNA gains and losses in primary endometrial carcinomas by comparative genomic hybridization, Genes Chromosomes Cancer 18(2) (1997), 115-125.

[37] R.G. Steinbeck, The DNA content of chromosome division figures and interphase nuclei classifies ulcerative colitis, Eur. J. Cancer 34(1) (1998), 175-181.

[38] P. Tan, B. Cady, M. Wanner, P. Worland, B. Cukor, C. MagiGalluzzi, P. Lavin, G. Draetta, M. Pagano and M. Loda, The cell cycle inhibitor p27 is an independent prognostic marker in small (T1a,b) invasive breast carcinomas, Cancer Res. 57(7) (1997), 1259-1263.

[39] M.M. Tanner, M. Tirkkonen, A. Kallioniemi, K. Holli, C. Collins, D. Kowbel, J.W. Gray, O.P. Kallioniemi and J. Isola, Amplification of chromosomal region 20q13 in invasive breast cancer: prognostic implications, Clin. Cancer Res. 1(12) (1995), 1455-1461.

[40] H. Tsuda, D.F. Callen, T. Fukutomi, Y. Nakamura and S. Hirohashi, Allele loss on chromosome 16q24.2-qter occurs frequently in breast cancers irrespectively of differences in phenotype and extent of spread, Cancer Res. 54(2) (1994), 513-517.

[41] M.L. Yaremko, W.M. Recant and C.A. Westbrook, Loss of heterozygosity from the short arm of chromosome 8 is an early event in breast cancers, Genes Chromosomes Cancer 13(3) (1995), 186-191. 


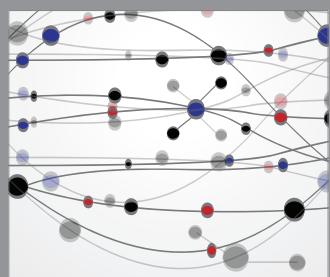

The Scientific World Journal
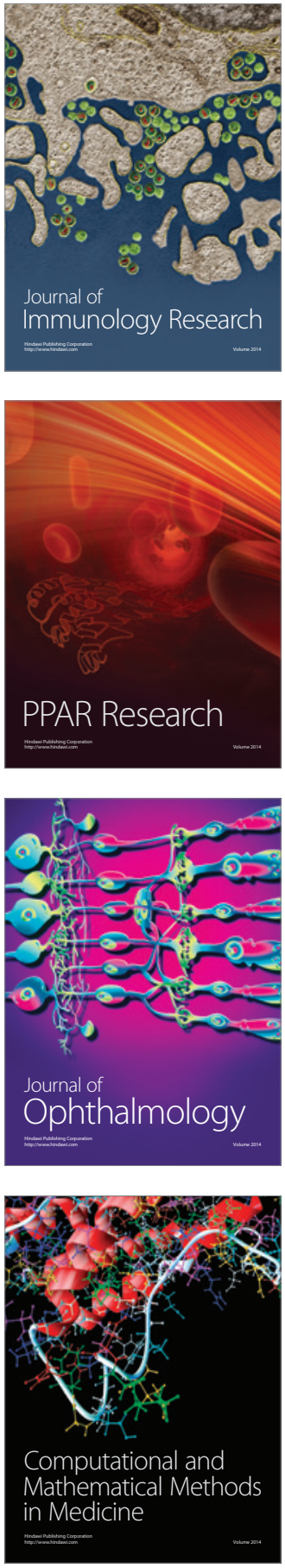

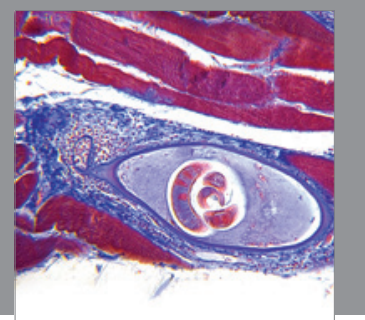

Gastroenterology

Research and Practice
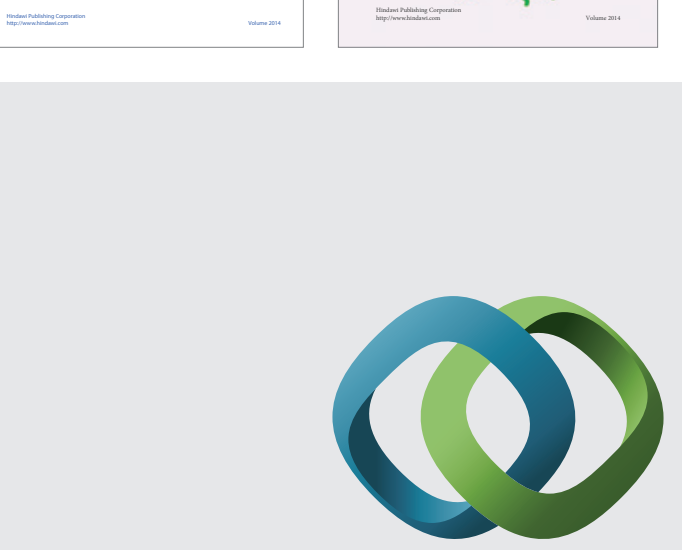

\section{Hindawi}

Submit your manuscripts at

http://www.hindawi.com
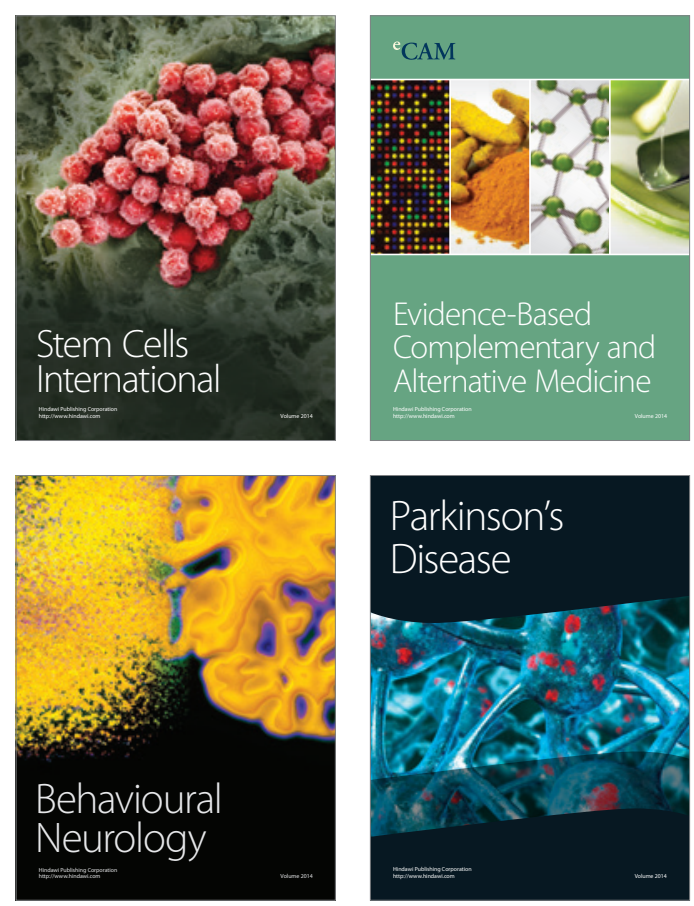

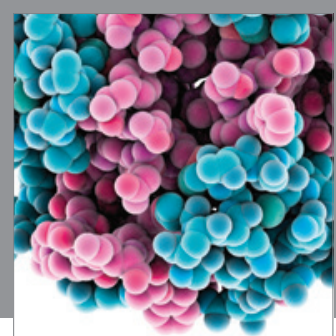

Journal of
Diabetes Research

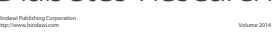

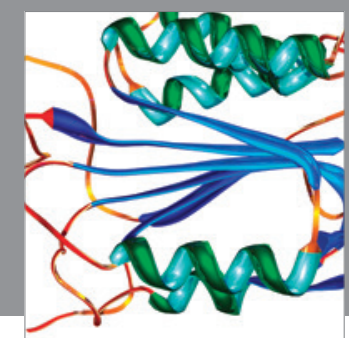

Disease Markers
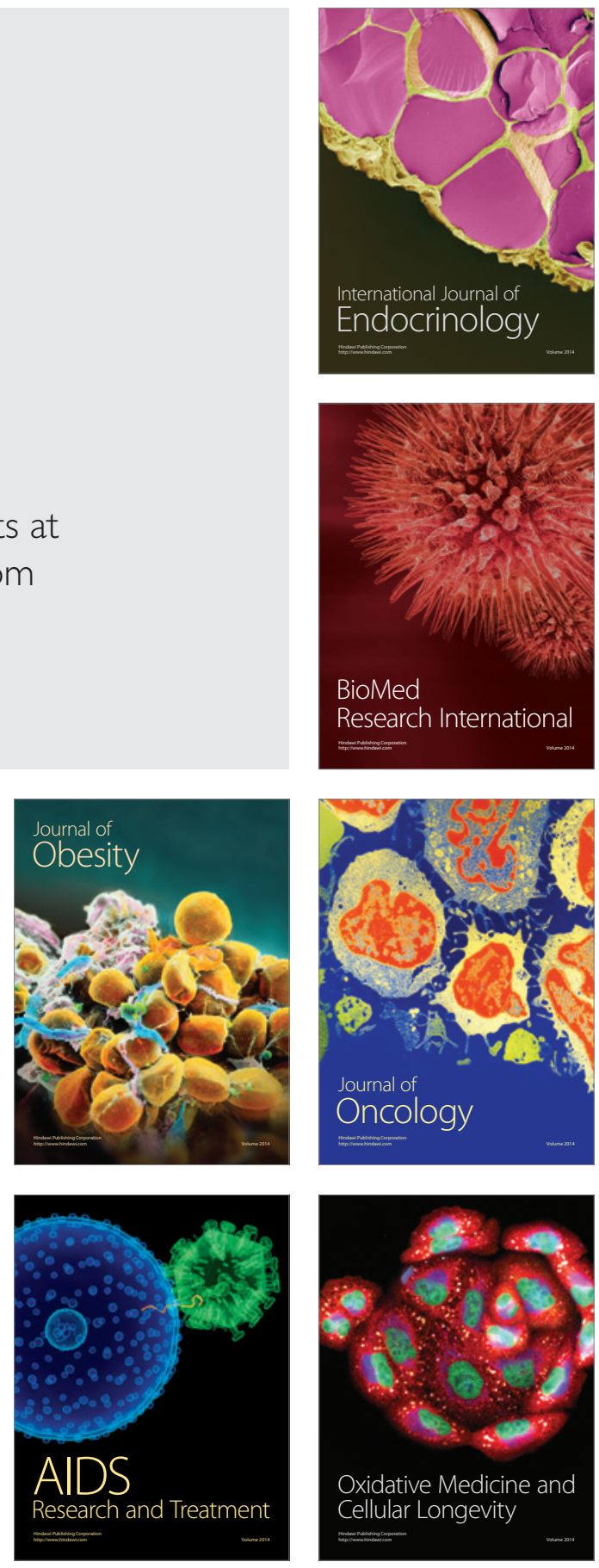